\title{
Racial disparities in perinatal mental health care during COVID-19
}

\section{The pandemic has exacerbated barriers to screening, diagnosis, and treatment of women of color}

$\mathrm{P}$ erinatal mental health disorders such as perinatal depression are common complications of pregnancy ${ }^{1}$ and cause significant disability in mothers and children. ${ }^{2}$ Yet despite facing higher 12-month rates of depression than White women, ${ }^{3}$ Black and Hispanic women are less likely than White women to be diagnosed with and receive treatment for postpartum depression. ${ }^{4}$

In addition to leading to $>800,000$ deaths in the United States alone (as of mid-December 2021), ${ }^{5}$ COVID-19 has disrupted health care delivery, including perinatal mental health services. ${ }^{6}$ Emerging data also describe neuropsychiatric effects of COVID-19 on both infected and uninfected individuals. ${ }^{7}$ Because Black and Hispanic individuals bear a disproportionate burden of COVID-19, ${ }^{8}$ compared to White women, women of color stand to be more adversely impacted by the direct effects of the disease as well as by related disruptions in perinatal psychiatry services.

Reasons for perinatal health disparities are multifactorial, complex, and interrelated. Disparities, which can be seen as proportionate differences in access by members of minority groups compared with groups in the majority, are related to differences in mental health screening, health care accessibility, and decisions to initiate treatment. In this commentary, we define "women of color" as non-White women, and focus on how traditional barriers to perinatal mental health treatment in women of color are exacerbated in the era of

\section{Disclosures}

The authors report no financial relationships with any companies whose products are mentioned in this article, or with manufacturers of competing products. The opinions expressed in this article are those of the authors and not of Duke University, Massachusetts General Hospital, or the US Government.

doi: $10.12788 /$ cp.0206

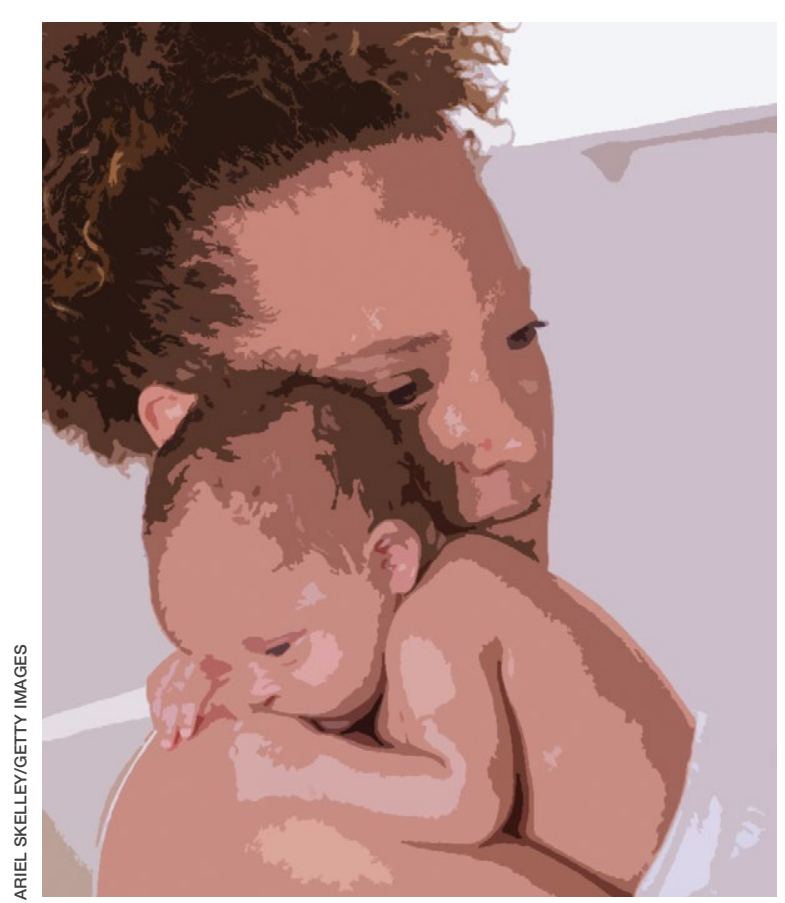

Nkechi Conteh, MBBS, MPH

Instructor in Psychiatry

Ammon-Pinizzotto Center for

Women's Mental Health

Department of Psychiatry

Massachusetts General Hospital

Harvard Medical School

Boston, Massachusetts

\section{Colin Smith, MD}

Chief Resident, Internal MedicinePsychiatry, PGY-6

Department of Psychiatry and

Behavioral Sciences

Department of Medicine

Duke University Hospital

Durham, North Carolina

\section{Cole Davis}

BSc Candidate in Chemistry

Trinity College of Arts and Sciences

Duke University

Durham, North Carolina
Marla Wald, MD

Associate Professor

Department of Psychiatry and

Behavioral Sciences

Duke University Hospital

Duke University School of Medicine

Durham, North Carolina

Jane Gagliardi, MD, MHS

Associate Professor

Department of Psychiatry and

Behavioral Sciences

Department of Medicine

Duke University Hospital

Duke University School of Medicine

Durham, North Carolina 


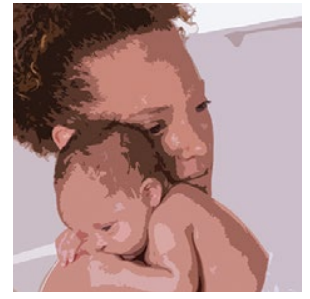

Perinatal mental health

\section{Clinical Point}

Black, Asian, and
American Indian
and Alaska Native
women are less likely
than White women
to be screened for
depression

f

Discuss this article at www.facebook.com/ MDedgePsychiatry
COVID-19. We focus primarily on postpartum depression because it is the peripartum mental health disorder with the highest likelihood of uptake in screening and treatment practices; however, disparities may be present in other mental health disorders during this period.

\section{Gaps in screening and identification}

Postpartum depression is a source of mitigatable risk for mother and neonate in the peripartum period, and the topic of screening for its presence arises in educational and best practices materials for primary care, OB-GYN, and pediatric care clinicians. Despite considerable evidence demonstrating better outcomes (for mother and child) with early detection and treatment of perinatal mental health disorders, racial and ethnic disparities persist in the screening process. At baseline, Black, Asian, and American Indian and Alaska Native women are less likely than White women to be screened for depression. ${ }^{9}$ Research shows that screening practices differ based on type of clinic, with one study noting that patients of family physicians were more likely to be screened for perinatal depression than were patients of OB-GYNs or nursing midwives. ${ }^{9}$ Even after adjusting for clinic type, racial differences in screening persist, with fewer women of color screened than their White counterparts. ${ }^{9}$ The literature consistently shows that within the same care settings, physicians deliver less information, less supportive talk, and less evidence-based treatment to Black and Hispanic patients and patients of lower economic status. ${ }^{10-12}$ Patient-clinician ethnic concordance is shown to positively impact the therapeutic relationship; at present, depressive symptoms are underrecognized in people of color, for whom referral to psychiatric care may be further compounded by inadequate knowledge of psychiatric resources. ${ }^{10-13}$

Data from Medicaid programs reveal that compared to White women, Black women are less likely to attend postpartum visits, which leads to a downstream effect on the ability to identify Black women with mental health disorders during the postpartum period. ${ }^{14}$ In addition to experiencing fewer opportunities for detection, women of color are more likely to report somatic symptoms of depression, which may not be detected in routinely employed perinatal depression screening tools..$^{15}$

\section{Disparities in accessibility and treatment}

Black women are more likely to present in crisis and, hence, to acute care settings, which is likely related to disparities in screening and early detection..$^{16,17}$ In a recent study investigating racial and ethnic differences in postpartum depression care, Chan et $\mathrm{al}^{16}$ found that Black women experience higher rates of hospital-based care compared with other racial groups. This study highlights the unavailability or inaccessibility of primary preventive measures to women in racial minority groups, which supports earlier studies that reported a correlation between access to care and severity of illness. ${ }^{16}$ Women in crisis may experience magnified disparities in access to high-quality care as they encounter institutional racism, potential loss of parental rights, and barriers due to insurance status. ${ }^{17,18}$ Furthermore, access to care for patients who are members of racial minority groups is limited in settings where culturally competent practices are absent or diminished, or discriminatory procedures are implicitly accepted and prevalent. ${ }^{12,19-22}$ The adverse impact of language constraints on accessibility of care is also well-documented, with recommendations such as ready access to interpreters to mitigate against miscommunications. ${ }^{23}$

Black and Hispanic women also experience significant delays between the time of delivery and treatment initiation. ${ }^{4}$ Studies of postpartum depression detection and treatment in specialty and primary care clinics show that, even when they desire treatment, women of color are less likely than White women to be offered treatment for postpartum depression. ${ }^{24}$ In terms of treatment options, research suggests women of color prefer psychotherapy over medication management. ${ }^{25,26}$ However, studies show that White women are more likely to be referred to psychotherapy. ${ }^{27}$ Research 
also reveals that Black and Hispanic women who are receptive to psychotropic medications have reduced rates of medication refills, ${ }^{4}$ which suggests that in these patients, counseling and monitoring adverse effects is suboptimal. In terms of treatment for substance use disorders (SUDs), after adjusting for maternal characteristics, Black and Hispanic women are significantly less likely to receive medication-assisted treatment (MAT) in pregnancy, ${ }^{28}$ and MAT is significantly less likely to be available in neighborhoods more densely populated by individuals of color. ${ }^{29,30}$

Several studies have explored possible explanations for discrepancies in treatment, including cultural expectations, differences in socioeconomic class, and racism. The stigma associated with psychiatric illness, misinformation about psychiatric treatments, and financial limitations have a substantial bearing on a patient's willingness or ability to engage in psychiatric care. ${ }^{25}$ Regarding SUDs, a fear of legal reprisal is likely to deter women of color from seeking care. ${ }^{31}$ Such fears are not unfounded; research has demonstrated that interactions with Child Protective Services are increased among women of color compared to White women in similar situations. ${ }^{32}$

Furthermore, there is evidence that women of color receive less practical support, such as childcare, breastfeeding support, and transportation, during the postpartum period. Despite the preponderance of literature demonstrating the psychological benefits of breastfeeding, , $^{33,34}$ structural and psychosocial barriers appear to disproportionately affect breastfeeding rates in Hispanic, Black, American Indian, and Native women, with Black women experiencing the lowest rates of breastfeeding overall. ${ }^{35}$ Women in minority groups additionally experience disproportionate uncertainty about employment-based breastfeeding regulations..$^{35,36}$ Specifically, many low-income jobs are not covered under the Family and Medical Leave Act, and compared to White women, Black women return to work on average 2 weeks earlier to jobs that are less welcoming to breastfeeding. ${ }^{35}$ In addition, insufficient education and support from health care settings and counselors play significant roles in disincentivizing women in minority groups from engaging in recommended breastfeeding and childcare practices. ${ }^{37,38}$

\section{COVID-19's influence on these disparities}

The COVID-19 pandemic has disproportionately impacted individuals of color. Black communities have experienced a higher rate of COVID-19 infection and a higher rate of death attributed to COVID19 , even after adjusting for age, poverty, medical comorbidities, and epidemic duration. ${ }^{39}$ The reasons for the disproportionate effects of the pandemic are complex and deeply ingrained in society. ${ }^{39}$ Emerging data indicate that COVID-19 might also lead to increased levels of psychological distress, anxiety, and depression in pregnant women $^{33,40,41}$ and in Black women in particular. ${ }^{42}$ A survey of 913 pregnant women in Philadelphia conducted in May 2020 found significantly higher rates of anxiety and depression among Black women compared with White women, even after controlling for maternal age, gestational age, socioeconomic status, and marital status..$^{42}$ A crosssectional study of 163 women found that during the perinatal period, women of color were more likely than their White counterparts to experience negative changes in their mental health. ${ }^{43}$ These differences are concerning because pregnant women who experience high levels of stress during the pandemic are at high risk for preterm delivery and perinatal complications. ${ }^{44}$

Women of color may be disproportionately excluded by models of care that have become commonplace during the pandemic. Remote obstetric care became more common during the COVID-19 pandemic ${ }^{45}$; however, Black and Hispanic patients have been less likely than White patients to use telehealth services. ${ }^{46}$ Whether the differences are related to a lower likelihood of having a usual source of care, less access to digital resources, decreased awareness of the availability of telehealth, or less familiarity with digital technology, the common factor in all of the hypothesized reasons is structural racism. ${ }^{46}$ This is despite the fact

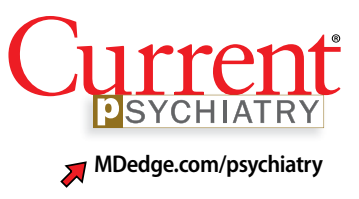

Clinical Point

Women of color

receive less

practical support

such as childcare

and breastfeeding

support during the

postpartum period 


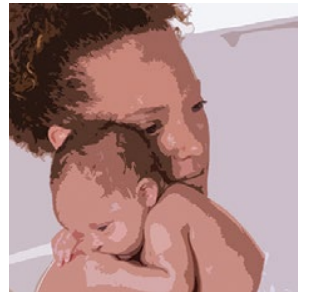

Perinatal mental health

\section{Clinical Point}

Teleheath became more common during COVID-19, but Black and Hispanic patients are less likely than White patients to use telehealth services that pregnant Black women report higher rates of concern than their White peers regarding the quality of their prenatal care during the pandemic. ${ }^{42}$ In a small study that surveyed 100 women about their preference for obstetric care, a significantly higher proportion of White women preferred virtual visits, with non-White women preferring in-person visits. ${ }^{47}$ Reasons cited for preferring virtual visits included convenience, safety with respect to viral transmission, compatibility with working from home, and less time waiting for the clinician; reasons cited for preferring in-person visits included a feeling of missing out on important parts of care, receiving less clinician attention, and having less of a connection with their clinician during virtual visits. ${ }^{47}$ Women of color have lower rates of perinatal depression screening than their White counterparts, ${ }^{9}$ and less frequent telehealth visits might lead to a further reduction in the detection and treatment of depression and other mental health conditions in this population.

Along with increasing telehealth services during the pandemic, many hospitals implemented stricter visitation policies for patients, including women giving birth, with the potential for greater detrimental impact on women of color. Before the pandemic, a survey of $>2,500$ women found that up to $10 \%$ of Black women reported experiencing racism during hospitalization for obstetrics-related care. ${ }^{48}$ These women also reported barriers to open and supportive communication with their clinicians. ${ }^{48} \mathrm{~A}$ recent study by Gur et $\mathrm{al}^{42}$ found that pregnant Black women reported more worries about the birthing experience during the pandemic than White women. In a setting with restricted visitors, all women are at risk for having a lonelier birth experience, but women of color who are already concerned about barriers to communication and racist care practices also must contend with their lived experience of systemic inequity, barriers to communication, and concerns about frank racism, without the support and potential advocacy they may usually rely upon to get them through medical experiences. Furthermore, pregnant women with mental illness are at greater risk for pregnancy complications. Together, these data suggest that women in minority groups who are pregnant and have mental illness are particularly vulnerable and are at greater risk without social support and advocacy during hospitalization.

The postpartum period is accompanied by unique concerns in terms of breastfeeding and social support for women of color. Women in minority groups had lower breastfeeding rates before the pandemic. Several studies looked at the impact of COVID-19 and associated restrictions on breastfeeding. In the United Kingdom, women in minority groups were more likely to stop breastfeeding due to the challenges of COVID-19-related restrictions. ${ }^{49}$ Compared with White women, these women were also more likely to report less practical support for breastfeeding during the pandemic. ${ }^{49}$ Other factors associated with low breastfeeding rates include lower levels of education and stressful living conditions. ${ }^{49}$ Though these factors were present before COVID-19, the pandemic has exacerbated these differences. Taken together, the evidence points to a role of long-standing structural and systemic inequity and racism in the health and wellbeing of women in minority groups.

\section{A look towards solutions}

Although perinatal mental health racial disparities predate the COVID-19 pandemic, differences in access to screening, identification, and treatment for mental health disorders place pregnant women of color and their children at heightened risk for poor health outcomes compared to their White counterparts during and after the pandemic. Despite the advent and progression of telehealth, existing race-based differences appear to have been maintained or exacerbated. The reasons for disparities are multifactorial and interrelated, and some of the outcomes perpetuate certain drivers of racism, which in turn drive continued inequity. Given the symptoms of depression, it is especially worrisome that clinicians may expect vulnerable women with illnessinduced amotivation, anhedonia, and apathy to advocate for their own care. 
Overall, the evidence confirms an imperative need-before, during, and after the COVID-19 pandemic - to provide education in mental health and cultural competency to clinicians such as obstetricians and pediatricians, who are more likely to have the first contact with women with perinatal depression. Health systems and government agencies also bear a responsibility to provide avenues for perinatal care clinicians to receive training and to increase access to culturally appropriate treatments through policy and structural changes.

\section{References}

1. Woody CA, Ferrari AJ, Siskind DJ, et al. A systematic review and meta-regression of the prevalence and incidence of perinatal depression. J Affect Disord. 2017;219:86-92.

2. Slomian J, Honvo G, Emonts P, et al. Consequences of maternal postpartum depression: a systematic review of maternal and infant outcomes. Womens Health (Lond). 2019;15:174550651984404.

3. Kurz B, Hesselbrock M. Ethnic differences in mental health symptomatology and mental health care utilization among WIC mothers. Social Work in Mental Health. 2006;4(3):1-21.

4. Kozhimannil KB, Trinacty CM, Busch AB, et al. Racial and ethnic disparities in postpartum depression care among lowincome women. Psychiatr Serv. 2011;62(6):619-625.

5. COVID-19 global cases. Coronavirus Resource Center for Systems Science and Engineering. Johns Hopkins University. Accessed December 10, 2021. https://coronavirus.jhu.edu/ map.html

6. Gressier F, Mezzacappa A, Lasica PA, et al. COVID outbreak is changing our practices of perinatal psychiatry. Arch Womens Ment Health. 2020;23(6):791-792.

7. Troyer EA, Kohn JN, Hong S. Are we facing a crashing wave of neuropsychiatric sequelae of COVID-19? Neuropsychiatric symptoms and potential immunologic mechanisms. Brain Behav Immun. 2020;87:34-39.

8. COVID-19: Data. NYC Health. Accessed February 3, 2021 https://www1.nyc.gov/site/doh/covid/covid-19-data. page

9. Sidebottom A, Vacquier M, LaRusso E, et al. Perinatal depression screening practices in a large health system: identifying current state and assessing opportunities to provide more equitable care. Arch Womens Ment Health. 2021;24(1):133-144.

10. Ma A, Sanchez A, Ma M. The impact of patient-provider race/ethnicity concordance on provider visits: updated evidence from the medical expenditure panel survey. J Racial Ethn Health Disparities. 2019;6(5):1011-1020.

11. Greenwood BN, Hardeman RR, Huang L, et al. Physicianpatient racial concordance and disparities in birthing mortality for newborns. Proc Natl Acad Sci USA. 2020; 117(35):21194-21200.

12. Chaudron LH, Kitzman HJ, Peifer KL, et al. Self-recognition of and provider response to maternal depressive symptoms in low-income Hispanic women. J Womens Health (Larchmt). 2005;14(4):331-338.

13. Institute of Medicine. Unequal treatment: confronting racial and ethnic disparities in health care. The National Academies Press; 2003. Accessed December 7, 2021. https:/ / www.nap. edu/catalog/12875/unequal-treatment-confronting-racialand-ethnic-disparities-in-health-care

14. Thiel de Bocanegra $H$, Braughton M, Bradsberry $M$, et al. Racial and ethnic disparities in postpartum care and contraception in California's Medicaid program. Am J Obstet Gynecol. 2017;217(1):47.e1-47.e7.

15. Nadeem E, Lange JM, Miranda J. Perceived need for care among low-income immigrant and U.S.-born Black and Latina women with depression. J Womens Health (Larchmt). 2009;18(3):369-375

16. Chan AL, Guo N, Popat R, et al. Racial and ethnic disparities in hospital-based care associated with postpartum depression. J Racial Ethn Health Disparities. 2021;8(1):220-229.

17. Kopelman R, Moel J, Mertens C, et al. Barriers to care for antenatal depression. Psychiatr Serv. 2008;59(4):429-432.

18. Kimerling R, Baumrind N. Access to specialty mental health services among women in California. Psychiatr Serv. 2005;56(6):729-734

19. Ta Park V, Goyal D, Nguyen T, et al. Postpartum traditions, mental health, and help-seeking considerations among Vietnamese American women: a mixed-methods pilot study. J Behav Health Serv Res. 2017;44(3):428-441.

20. Chen F, Fryer GE Jr, Phillips RL Jr, et al. Patients' beliefs about racism, preferences for physician race, and satisfaction with care. Ann Fam Med. 2005;3(2):138-143.

21. Holopainen D. The experience of seeking help for postnatal depression. Aust J Adv Nurs. 2002;19(3):39-44.

22. Alvidrez J, Azocar F. Distressed women's clinic patients: preferences for mental health treatments and perceived obstacles. Gen Hosp Psychiatry. 1999;21(5):340-347.

23. Lara-Cinisomo S, Clark CT, Wood J. Increasing diagnosis and treatment of perinatal depression in Latinas and African American women: addressing stigma is not enough. Womens Health Issues. 2018;28(3):201-204.

24. Zittel-Palamara K, Rockmaker JR, Schwabel KM, et al Desired assistance versus care received for postpartum depression: access to care differences by race. Arch Womens Ment Health. 2008;11(2):81-92.

25. Dennis CL, Chung-Lee L. Postpartum depression helpseeking barriers and maternal treatment preferences: a qualitative systematic review. Birth. 2006;33(4):323-331.

26. Cooper LA, Gonzales JJ, Gallo JJ, et al. The acceptability of treatment for depression among African American, Hispanic, and white primary care patients. Med Care 2003;41(4):479-489

27. House TS, Alnajjar E, Mulekar M, et al. Mommy meltdown: understanding racial differences between black and white women in attitudes about postpartum depression and treatment modalities. J Clin Gynecol Obstet. 2020;9(3):37-42.

28. Schiff DM, Nielsen T, Hoeppner BB, et al. Assessment of racial and ethnic disparities in the use of medication to treat opioid use disorder among pregnant women in Massachusetts. JAMA Netw Open. 2020;3(5):e205734.

29. Hansen H, Siegel C, Wanderling J, et al. Buprenorphine and methadone treatment for opioid dependence by income, ethnicity, and race of neighborhoods in New York City. Drug Alcohol Depend. 2016;164:14-21.

30. Goedel WC, Shapiro A, Cerdá M, et al. Association of racial/ ethnic segregation with treatment capacity for opioid use

\section{Bottom Line}

Racial disparities in perinatal mental health care persist despite widespread incorporation of telehealth into psychiatric services. Until causal factors are appropriately addressed through education, implementation, and structural changes, the benefits that have accompanied expanded psychiatric services via telehealth may only serve to exacerbate these differences. 


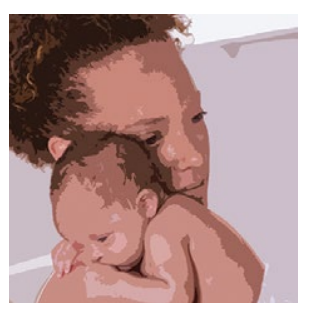

Perinatal mental health

\section{Clinical Point}

\section{Health systems and government agencies need to increase access to culturally appropriate treatments}

disorder in counties in the United States. JAMA Netw Open. 2020;3(4):e203711.

31. Stone R. Pregnant women and substance use: fear, stigma, and barriers to care. Health Justice. 2015;3:2.

32. Roberts SC, Nuru-Jeter A. Universal screening for alcohol and drug use and racial disparities in child protective services reporting. J Behav Health Serv Res. 2012;39(1):3-16.

33. Krol KM, Grossmann T. Psychological effects of breastfeeding on children and mothers. Bundesgesundheitsblatt Gesundheitsforschung Gesundheitsschutz. 2018;61(8): 977-985.

34. Evans K, Labbok M, Abrahams SW. WIC and breastfeeding support services: does the mix of services offered vary with race and ethnicity? Breastfeed Med. 2011;6(6):401-406.

35. Jones KM, Power ML, Queenan JT, et al. Racial and ethnic disparities in breastfeeding. Breastfeed Med. 2015;10(4): 186-196.

36. Hohl S, Thompson B, Escareño M, et al. Cultural norms in conflict: breastfeeding among Hispanic immigrants in rural Washington state. Matern Child Health J. 2016;20(7): 1549-1557.

37. McKinney CO, Hahn-Holbrook J, Chase-Lansdale PL, et al. Racial and ethnic differences in breastfeeding. Pediatrics. 2016;138(2):e20152388.

38. Louis-Jacques A, Deubel TF, Taylor M, et al. Racial and ethnic disparities in U.S. breastfeeding and implications for maternal and child health outcomes. Semin Perinatol. 2017;41(5):299-307.

39. Millett GA, Jones AT, Benkeser D, et al. Assessing differential impacts of COVID-19 on black communities. Ann Epidemiol. 2020;47:37-44.

40. Fan S, Guan J, Cao L, et al. Psychological effects caused by COVID-19 pandemic on pregnant women: a systematic review with meta-analysis. Asian J Psychiatr. 2021;56:102533.
41. Robinson GE, Benders-Hadi N, Conteh N, et al. Psychological impact of COVID-19 on pregnancy. J Nerv Ment Dis. 2021;209(6):396-397.

42. Gur RE, White LK, Waller R, et al. The disproportionate burden of the COVID-19 pandemic among pregnant Black women. Psychiatry Res. 2020;293: 113475.

43. Masters GA, Asipenko E, Bergman AL, et al. Impact of the COVID-19 pandemic on mental health, access to care, and health disparities in the perinatal period. J Psychiatr Res. 2021;137:126-130.

44. Preis H, Mahaffey B, Pati S, et al. Adverse perinatal outcomes predicted by prenatal maternal stress among U.S. women at the COVID-19 pandemic onset. Ann Behav Med. 2021;55(3):179-191.

45. Fryer K, Delgado A, Foti T, et al. Implementation of obstetric telehealth during COVID-19 and beyond. Matern Child Health J. 2020;24(9):1104-1110.

46. Weber E, Miller SJ, Astha V, et al. Characteristics of telehealth users in NYC for COVID-related care during the coronavirus pandemic. J Am Med Inform Assoc. 2020;27(12):1949-1954.

47. Sullivan MW, Kanbergs AN, Burdette ER, et al. Acceptability of virtual prenatal care: thinking beyond the pandemic. J Matern Fetal Neonatal Med. 2021:1-4.

48. National Partnership for Women \& Families. Listening to Black mothers in California. Issue Brief. September 2018. Accessed December 7, 2021. https://www. nationalpartnership.org/our-work/resources/health-care/ maternity/listening-to-black-mothers-in-california.pdf

49. Brown A, Shenker N. Experiences of breastfeeding during COVID-19: lessons for future practical and emotional support. Matern Child Nutr. 2021;17(1): e13088. 